International Journal of Constructive Research in Civil Engineering (IJCRCE)

Volume 5, Issue 1, 2019, PP 24-30

ISSN 2454-8693 (Online)

DOI: http://dx.doi.org/10.20431/2454-8693.0501004

www.arcjournals.org

\title{
The Influence of Rock Anisotropy on the Plan of Constructions
}

\author{
Moein Zargar ${ }^{1}$, Hossein Gholami ${ }^{2}$, Hossein Norouzi ${ }^{3}$, Morteza Soltani ${ }^{4}$, Shahide Dehghan ${ }^{5}$, \\ Vijay P. Singh ${ }^{6}$, Mohsen Ghane ${ }^{7 *}$, Kaveh Ostad-Ali-Askari ${ }^{2}$ \\ ${ }^{I}$ Department of Civil Engineering, Farzanegan Institute of Higher Education, Isfahan, Iran \\ ${ }^{2}$ Department of Civil Engineering, Isfahan (Khorasgan) Branch, Islamic Azad University, Isfahan, Iran \\ ${ }^{3}$ Department of Civil Engineering, Maybod Branch, Islamic Azad University, Maybod, Yazd, Iran \\ ${ }^{4}$ Department of Architectural Engineering, Shahinshahr Branch, Islamic Azad University, Shahinshahr, Iran \\ ${ }^{5}$ Department of Geography, Najafabad Branch, Islamic Azad University, Najafabad, Iran \\ ${ }^{6}$ Department of Biological and Agricultural Engineering \& Zachry Department of Civil Engineering, Texas A \\ and M University, 321 Scoates Hall, 2117 TAMU, College Station, Texas 77843-2117, U.S.A. \\ ${ }^{7}$ Civil Engineering Department, South Tehran Branch, Islamic Azad University, Tehran, Iran
}

*Corresponding Author: Mohsen Ghane, Civil Engineering Department, South Tehran Branch, Islamic Azad University, Tehran, Iran

\begin{abstract}
A general condition for the strength standard of regular jointed rocks is obtained in this article. The procedure is depend on the performance of the homogenization procedure of periodic media within the framework of the yield plan principles. A rigorous explicit statement of the macroscopic standard is given as a sunordinate of the strongest features of the virgin rock and of the joints shaped as ports. Consideration is concentrated on the special case of an endlessly persistence entire rock. It is displayed in specific how the related velocity jumps for the homogenized medium can be determined from the presentation of the set of permissible stress vectors acting upon an elementary surface. This contains issues such as the significations of the intermittent nature of rock masses, extent states and failure modalities, strength standard, characteristic quantities and partial components for rock mass components, rock mass features, use of classification systems in plan, among the most significant issues. Anisotropy in the physical and mechanical behavior of the rocks is an effective parameter in determination of their engineering characteristics. The sedimentary and metamorphic rocks show anisotropic behavior due to higher lamination, bedding and flakiness. In this paper, the anisotropic behavior of clay rocks such as shale has been investigated. These types of rocks arise a lot of problems for engineers in designing the associated structures. The most important characteristic of this type of rocks is that they are composed of ultra-fine clay and silt particles. The discontinuities, fissures, and bedding layers and lamination in the shale masses change the mechanical characteristics and anisotropic behavior of these rock masses against the applied forces. Here, the anisotropic behavior of these types of rocks and the effect of different parameters and changes due to anisotropy are discussed.
\end{abstract}

Keywords: rock, construction, Anisotropy, plan

\section{INTRODUCTION}

Compressive strength of rocks is the most common parameter used in the stone engineering. In conventional engineering calculations and analyzes, rock is often considered as a linear elastic, homogeneous and isotropic material. While some of the rocks often show a particular physical and mechanical behavior that cannot be ignored in the accurate and complete solution of their behavior. A well-known behavior of such rocks that is revealed in many practical works is their anisotropic physical and mechanical behavior. Engineering activities such as construction of the underground structures including tunnels, open pit mines, dam foundations and, most importantly, evaluation of rock slopes requires accurate knowledge of the site and the physical and mechanical properties of the rocks which will be faced during the operation as well as determination of the tectonic conditions. Before any engineering operation, specific studies on the rock materials in the site should be done in accordance with the scope of the study. [5] There have been several basic expansions in rock 
engineering concentrate on new engineering tools for the analysis and design of structures in rock. One early enlargement was the origination of rock mass category systems which, when composed with experimental rules, prepares a framework that is still extensively applied for a plan. Another enlargement, relatively fuelled by an increment in computer power, is the multiplication of mathematical procedures that are routinely used to the resolution, an embodiment of the consequences, of multiplex 2D and 3D issues. Alas, our comprehending of doubt and originals of faults in rock engineering does not look to have been expanded to the same degree as these plan methods. Here, consideration is possessed to the originals of fault and doubt within these methods, and how these might harmfully influence our plans and administration of rock engineering plans. We deduce by offering that rock engineering, exclusively given the instruments and methods presently existing and under enlargement, would advantage from an essential endeavor to right measure and operate doubt and originals of error. According to the experiments, the failure condition of the samples is controlled by the degree of lamination and flakiness of the sample as well as the angle of flakiness and lamination relative to the loading direction on the sample. To study the effect of this angle (discontinuity angle), the stone coring was carried out in different directions between 0 and 90 degrees with 15degree intervals [5].

\section{METHODS}

The Mohr-Coulomb standard requires to be improved for especially anisotropic rock material and jointed rock masses. Taking $\sigma 2$ into account, a recent strength standard is proposed because both $\sigma 2$ and $\sigma 3$ would donate to the normal stress on the available plane of debility. This standard describes the increment of strength $(\sigma 2-\sigma 3)$ in the underground spouts because $\sigma 2$ along the tunnel axis is not quited notably. Other reason for stability increase is less decrease in the mass modulus in tunnels because of restricting dilatancy. Experimental relationships acquired from information from block shear experiments and uniaxial jacking experiments have been offered to assess recent stability variables. A relationship for the elastic stability of the rock mass is stated. Lastly, Hoek and Brown opinion is enlarged to calculate for $\sigma 2$. A usual stability standard for both based basement sprouts and rock gradients is offered. The penetration of anisotropy of these rocks on the sliding area is significantly decreased, and plastic-type rocks incite rock flow extension, which is generally later converted into multiplex landslides with prevailing revolutionary motion. It is displayed that the treatment of many such rocks can be described theoretically applying an analysis, first designed by Biot (1965b), of the treatment of a biotype, anisotropic frame. The definition of this anisotropy and its significance in governing the procedure in which the material contorts are considered. The primary enlargement of internal structures within a block of a homogeneous, anisotropic material is considered. It is deduced that rocks which have anisotropic stress/strain characteristics may become impermanent internally when pressed. This inconstancy, which does not presently include a qualification difference, dues to the expansion of inner constructions whose form is mostly determined by the degree of anisotropy of the rock. imagined as a possible intermittent to the classical design procedures utilized for analyzing the consistency of jointed rock structures, the homogenization procedure prescribed in this donation stems from the instinctive opinion that, from a macroscopic point of view, a rock mass crossed by a network of joint surfaces may be comprehended as a homogeneous medium. The stability standard of the latter can be theoretically defined from the knowledge of the failure conditions of the individual components, namely, rock matrix and joint junctions.

\section{RESULT AND DISCUSSIONS}

The investigation forcefully offers that loss to clay rock presenting on the gallery front donates to a consideration rise in the anisotropy ratio of the hydric strains. The Rock Quality Designation index is outlined as the percentage of the borehole core or scanline that contains whole lengths $\geq 0.1 \mathrm{~m}$, and has been widely applied as an engineering measure of the junction degree of a rock mass. The directionality of Rock Quality determination, which signifies the value of the Rock Quality Determination gained from drilling cores or scanlines with various directions can be varied for a given place, is generally considered as a drawback by scholars and inventors. This article shows a recent landscape for the directionality of Rock modality determination. The directionality of Rock Quality determination should be considered as an advantage of the index because it basically reverberates the anisotropy of rock masses. Since a smaller value of the Rock Quality determination index points a better duplication of the various jointing degrees of various rock masses, its minimum value is chosen 
as the significant Rock Quality determination, and its corresponding administration is named as the significant orientation of Rock Quality determination. In this article, to acquire the Rock Quality Determination and its significant orientation, theoretical formulas are obtained to compute the Rock Quality Determination in any orientation depend on some known Rock Quality Determinations in various orientations. The era of Structural Geology has noted a great enlargement in the explanation, explication, and modeling of distortion structures. Amongst components that qualify distortion and the resulting structures, mechanical anisotropy has proven hard to deal with. The consequences show that the peak shear stress was more sentient to the inclination angles than the normal stress. Rock bolting has been extensively used for amplifying basement openings, like tunnels and mining. The sensibility of anisotropic characteristics and the picture noise effect are better prospected to focus on the validity of the presented way for assessing the anisotropy of rock fabrics. When building diggings, the metameric facing installed in an anisotropic rock mass is sensitive to asymmetrical pressure and essential local inconsistency. A series of physical model experiments were executed to read the result of this maintenance system.
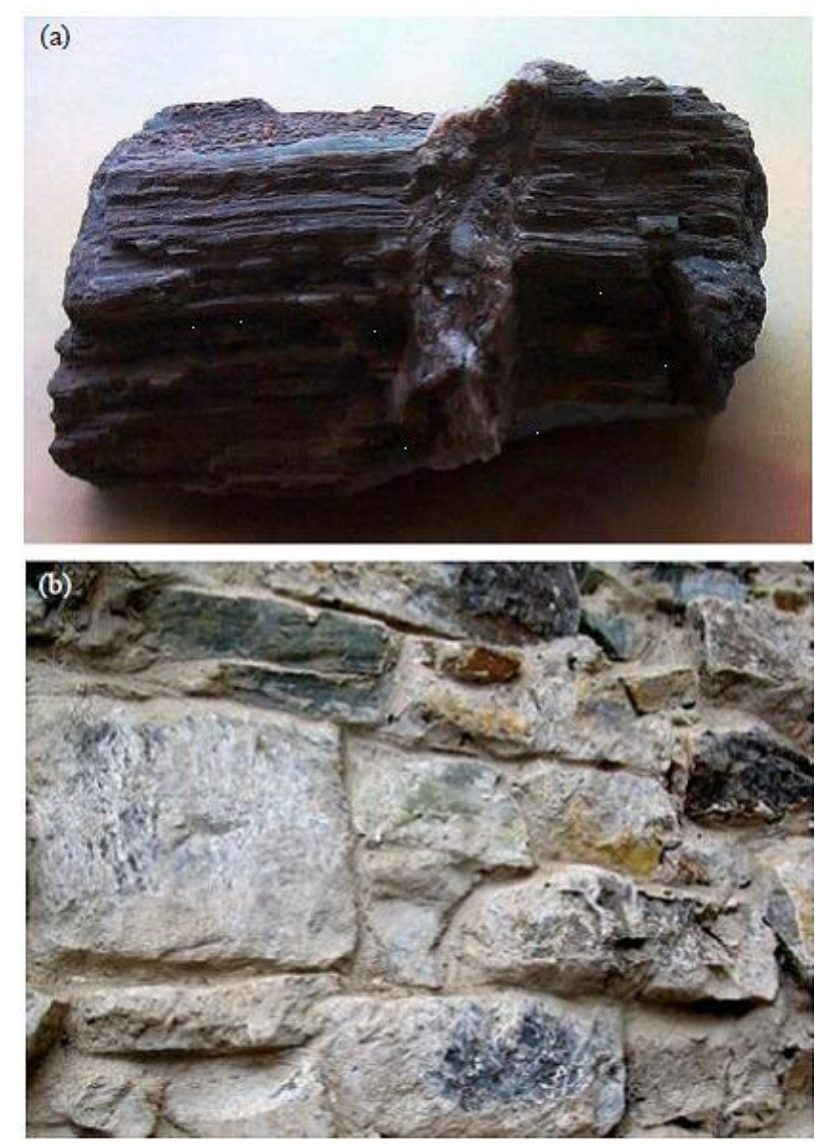

Fig1. Artificial material and its natural analogue: a) the crack of pushing in layered sandstone filled with feldspar and $b$ ) old masonry (Germany)

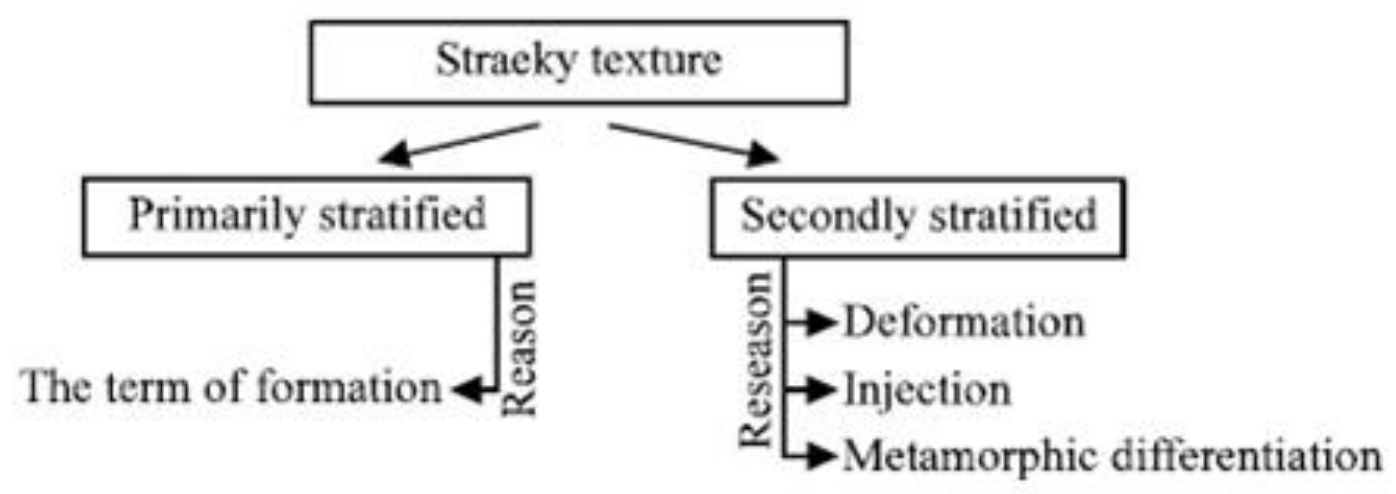

Fig2. Terms of creating anisotropy of physical and mechanical properties of rocks. 


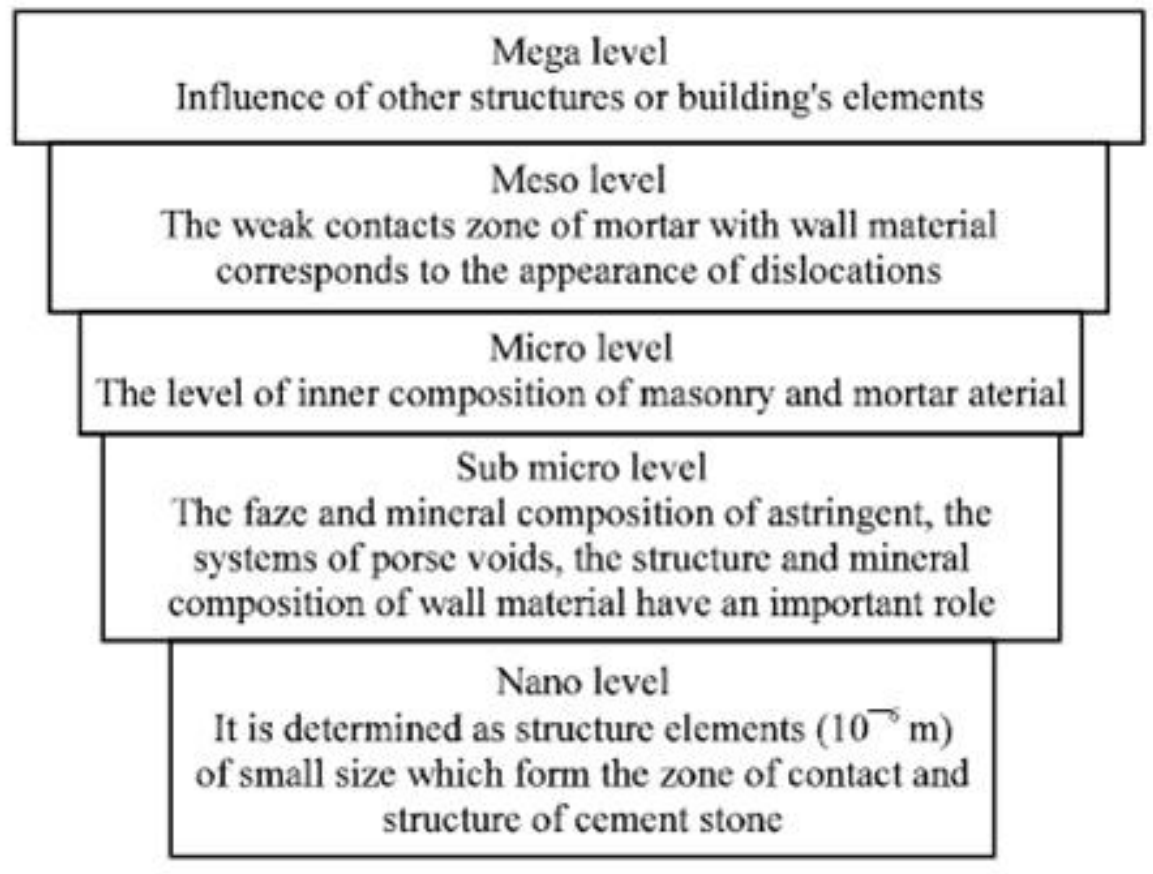

Fig3. Hierarchical system of intermittent and continuous cracks in the material.

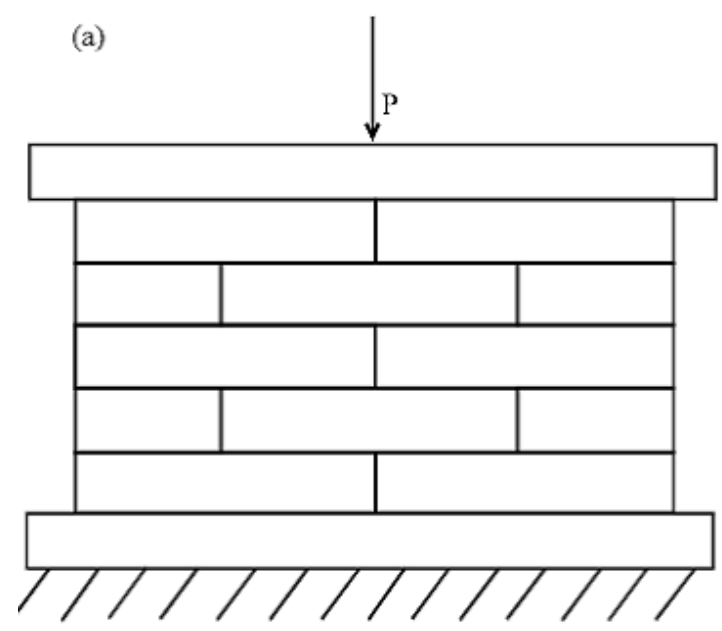

(b)

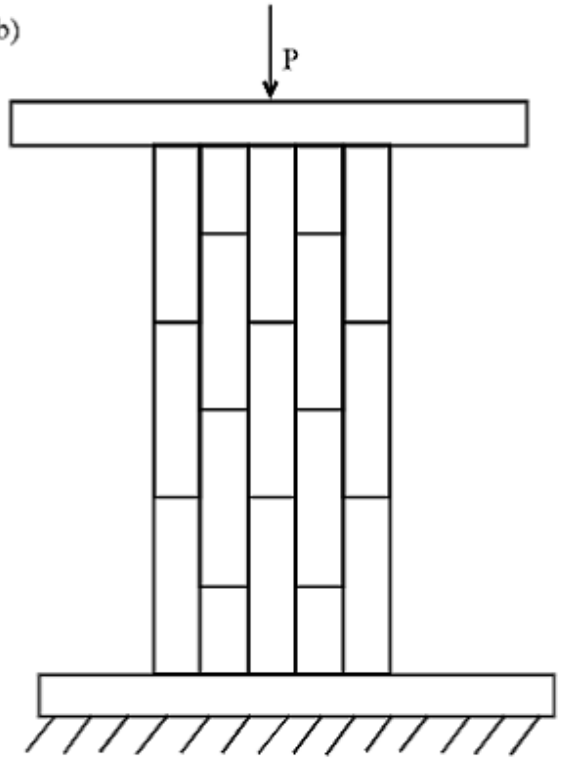

Fig4. Scheme of testing masonry samples by compressive load: a) perpendicular to the horizontal mortar seams and $b)$ parallel to horizontal mortar seams. 


\section{CONCLUSION}

According to the obtained results and considering the studies that have been carried out by different researchers on the metamorphic rocks, especially different types of schists and slate (Donath, 1964; Sing, 1988, Ghafoori M, 1996), the following results were obtained:

1. Anisotropic rocks show the maximum compressive strength in a direction often perpendicular and sometimes parallel to the lamination and flakiness. For this type of rock, the minimum resistance often occurs for an angle of 30 to 45 degrees between the anisotropic plane and direction of the maximum principal stress. The weak (lamination and schistosity) direction versus the maximum principal stress curve is concave upward. The maximum ultimate compressive strength was observed for 0 and 90 degrees and the minimum compressive strength was observed for about 30 degrees. The anisotropy degree that is defined as the maximum to the minimum compressive strengths ratio varied from 3 to 10 .

2. Under normal triaxial test conditions, the anisotropy effect decreased with the confining pressure.

3. With increasing the confining pressure, the compressive strength also increased, which was especially evident for samples with anisotropy parallel to the maximum principal stress direction.

4. It was observed from the experiments that the failure of the samples was controlled by the degree of lamination or flakiness as well as the angle of lamination relative to sample loading direction. Failure of such anisotropic rocks for angles of 0 to 15 degrees usually occurs in the cracks originating from weak surfaces, and for 15 to 45 degrees, or sometimes 60 degrees, failure occurs in the form of a slip on the existing weak surface (s). Finally, for angles of 60 to 90 degrees, the failure occurs in the form of the shear fracture in a direction of 20 to 30 degrees relative to the maximum stress direction. For samples with weak lamination, the effect of lamination on the sample failure was practically insignificant, so that the failure occurred in all the directions in the form of shear fracture.

5. The experimental results showed good agreement with the experimental criterion suggested by Jaeger (1960), called the Single Plane of Weakness Theory, and another criterion called Changes in the Friction Coefficient and Cohesion, which was later revised by Mclamore and Gray (1967).

6. The maximum and minimum longitudinal and lateral deformability of the anisotropic samples under the applied pressure was observed in the perpendicular and parallel directions to the anisotropic planes, respectively, which usually correspond to the minimum and maximum values of the Young modulus, respectively.

Geotechnical plan was slower than building plan in the usage of likely or semi-probabilistic methods. Testimonies for this may be the profound bases of experimental procedures applied in the plan of buildings in or on the land. The geotechnical plan does not consider made substances, with fairly familiar component values, but with natural substances, of a major variety as regards their source and the situation in which they are found in essence. Geotechnical buildings are not as well determined geometrically as the maker of a construction or a bridge, and the practices on them are also more hard to appoint and measure.

\section{REFERENCES}

[1] Donath, F.A.; 1964- "Strength variation and deformational behavior of anisotropic rocks. State of Stress in the Earth's crust, W.R Jude (editor)". American Elsevier Publishing Co., New York, N.Y., PP. 281-297. M.

[2] Mclamore, R., and Gray, K.E.; 1967- "The mechanical behavior of anisotropic sedimentary rocks", Trans, American Soci. Mech. Engrs., Series B, Vol. 89, PP. 62-76.

[3] Jaeger, J.C.; 1960- "Shear failure of anisotropic rocks", Geological Mag., Vol.97, PP. 65-72.

[4] Sing J.; 1988- "Strength prediction of anisotropic rocks". PhD Thesis, Indian Institute of Technology, New Delhi.

[5] Ghafoori M, 1996. "The effect of rock Anisotropy on the design of structures "Geological Society of IRAN"

[6] Jun Zheng, Xiaojuan Yang, et al. 2018. A new perspective for the directivity of Rock Quality Designation (RQD) and an anisotropy index of jointing degree for rock masses. Engineering Geology, Volume 240, 5 June 2018, Pages 81-94. 
[7] Huafeng Sun, Sandra Vega, Guo Tao, 2017. Analysis of heterogeneity and permeability anisotropy in carbonate rock samples using digital rock physics. Journal of Petroleum Science and Engineering, Volume 156, July 2017, Pages 419-429.

[8] Stephen Hedan, Valéry Valle, Justo Cabrera, Philippe Cosenza, 2018. A new approach to quantify the anisotropy of hydro mechanical strains in clay-rock at the gallery scale. International Journal of Rock Mechanics and Mining Sciences, Volume 111, November 2018, Pages 45-53.

[9] Peitao Wang, Meifeng Cai, Fenhua Ren. Anisotropy and directionality of tensile behaviours of a jointed rock mass subjected to numerical Brazilian tests. Tunnelling and Underground Space Technology, Volume 73, March 2018, Pages 139-153

[10] Hedan S, Valle V, et al, 2018. A new approach to quantify the anisotropy of hydromechanical strains in clay-rock at the gallery scale. International Journal of Rock Mechanics and Mining Sciences. Volume 111, November 2018, Pages 45-53. https://doi.org/10.1016/j.ijrmms.2018.07.004.

[11] Ran H, Riese T, et al. 2018. Time for anisotropy: The significance of mechanical anisotropy for the development of deformation structures. Journal of Structural Geology, Available online 20 May 2018. https://doi.org/10.1016/j.jsg.2018.04.019.

[12] Berčáková A, Melichar R, et al. 2017. Evaluation of Anisotropy of Fracture Toughness in Brittle Rock, Migmatized Gneiss. Procedia Engineering. Volume 191, 2017, Pages 900-907. https://doi.org/10 .1016/j.proeng.2017.05.260.

[13] H.Hatzor Y, Feng X, et al. 2015. Tunnel reinforcement in columnar jointed basalts: The role of rock mass anisotropy. Tunnelling and Underground Space Technology. Volume 46, February 2015, Pages 1-11.

[14] Wang P, Ren F, et.al. Evaluation of the anisotropy and directionality of a jointed rock mass under numerical direct shear tests. Engineering Geology. Volume 225, 20 July 2017, Pages 29-41.

[15] H.Kruhl J, 2013. Fractal-geometry techniques in the quantification of complex rock structures: A special view on scaling regimes, in homogeneity and anisotropy. Journal of Structural Geology. Volume 46, January 2013, Pages 2-21. https://doi.org/10.1016/j.jsg.2012.10.002.

[16] Özbek A, Gül M, et al. Anisotropy effect on strengths of metamorphic rocks. Journal of Rock Mechanics and Geotechnical Engineering. Volume 10, Issue 1, February 2018, Pages 164-175. https://doi.org/10 .1016/j.jrmge.2017.09.006.

[17] MinGao Liang Z, et al. 2019. An equivalent anchoring method for anisotropic rock masses in underground tunneling. Tunnelling and Underground Space Technology. Volume 85, March 2019, Pages 294-306.

[18] SupYun T, Jong Jeong Y, et.al. 2013. Evaluation of rock anisotropy using 3D X-ray computed tomography. Engineering Geology. Volume 163, 19 August 2013, Pages 11-19. https://doi.org/10 .1016/j.enggeo.2013.05.017.

[19] Hu, X, Fang Fang Y, et al. 2019. Analysis of the behavior of a novel support system in an anisotropic ally jointed rock mass. Tunnelling and Underground Space Technology. Volume 83, January 2019, Pages 113134.

[20] Zhang C-L, Armand G, et.al. 2019. Investigation on anisotropy of mechanical properties of CallovoOxfordian claystone. Engineering Geology. Volume 251, 9 March 2019, Pages 128-145. https://doi.org/10.1016/j.enggeo.2019.02.008.

[21] Lu Y, Xiao S, et al. 2019. Experimental study on rock-breaking performance of water jets generated by self-rotatory bit and rock failure mechanism. Powder Technology. Volume 346, 15 March 2019, Pages 203-216.

[22] KrausL, Kopeček J, 2019. Surface anisotropy and spin wave resonance in submicron amorphous wires. Journal of Magnetism and Magnetic Materials. Volume 473, 1 March 2019, Pages 284-290. https://doi.org/10.1016/j.jmmm.2018.10.045.

[23] Lu Z, 2019. Uppermost mantle velocity and anisotropy structure beneath the North China Craton and its adjacent regions. Tectonophysics. Volume 754, 5 March 2019, Pages 45-55. https://doi.org/ 10.1016/j.tecto.2019.01.014.

[24] Zhang F, Xiong Y, et al, 2019. Thermo-elasto-viscoplastic mechanical behavior of manmade rock and its numerical modeling. Underground Space. Available online 8 February 2019. https://doi.org/10 .1016/j.undsp.2018.12.003.

[25] Ding P, Di B, et al, 2018. P- and S-wave velocity and anisotropy in saturated rocks with aligned cracks. Wave Motion. Volume 81, September 2018, Pages 1-14.

[26] Wang J, /zhang F, et al.2019. Anisotropy in small-strain shears modulus of permafrost at rising temperatures. Cold Regions Science and Technology. Volume 160, April 2019, Pages 1-12. https://doi.org/10.1016/j.coldregions.2019.01.003.

[27] Yan J, Fan J, et al.2018. Rock fabric and pore structure of the Shahejie sandy conglomerates from the Dongying depression in the Bohai Bay Basin, East China. Marine and Petroleum Geology. Volume 97, November 2018, Pages 624-638. https://doi.org/10.1016/j.marpetgeo.2018.07.009. 
[28] Zeng L, Zhao J, et al. 2008. Impact of rock anisotropy on fracture development. Progress in Natural Science. Volume 18, Issue 11, 10 November 2008, Pages 1403-1408. https://doi.org/10.1016 /j.pnsc.2008.05.016.

[29] Tsang Ch-Fu, Bernier F, Davies C, 2005. Geohydromechanical processes in the Excavation Damaged Zone in crystalline rock, rock salt, and indurated and plastic clays-in the context of radioactive waste disposal. International Journal of Rock Mechanics and Mining Sciences. Volume 42, Issue 1, January 2005, Pages 109-125. https://doi.org/10.1016/j.ijrmms.2004.08.003.

[30] Maghous S, de Buhan P, Bekaert A. 1999.Failure design of jointed rock structures by means of a homogenization approach. Mechanics of Cohesive-frictional Materials banner..Wiley Online Library. Volume3, Issue3. July 1998. Pages 207-228

[31] Single B, Goel R.K., et al. 1998. Effect of intermediate principal stress on strength of anisotropic rock mass. Tunnelling and Underground Space Technology. Volume 13, Issue 1, January-March 1998, Pages 71-79. https://doi.org/10.1016/S0886-7798 (98)00023-6.

[32] A. M. Hefny, K. Y. Lo, 1999. Analytical solutions for stresses and displacements around tunnels driven in cross-anisotropic rocks. Wiley Online Library. International Journal for Numerical and Analytical in Geomechanics. Volume23, Issue2. February 1999. Pages 161-177. https://doi.org/10.1002/(SICI)10969853(199902)23:2<161::AID-NAG963>3.0.CO;2-B.

[33] Margielewsk W, 2006. Structural control and types of movements of rock mass in anisotropic rocks: Case studies in the Polish Flysch Carpathians. Geomorphology. Volume 77, Issues 1-2, 15 July 2006, Pages 4768. https://doi.org/10.1016/j.geomorph.2006.01.003.

[34] Gercek H, 2007. Poisson's ratio values for rocks..International Journal of Rock Mechanics and Mining Sciences Volume 44, Issue 1, January 2007, Pages 1-13. https://doi.org/10.1016/j.ijrmms.2006.04.011.

[35] Cobbold P.R., Cosgrove J.W., Summers J.M.1971. Development of internal structures in deformed anisotropic rocks. Tectonophysics. Volume 12, Issue 1, July 1971, Pages 23-53.

[36] Aleksandrovna Kuprina A, Stanislavovich Lesovik V,et al. 2014.Anisotropy of material properties of natural and Man-Triggered Origin. Research Journal of Applied Science 9 (11):816-819, 2014.ISSN: 1815-932X.

[37] Arora V.K., 1987. Strength and deformational behaviour of jointed rocks. A thesis Submitted in fulfilment of requirement for degree of Doctor of Philosophy. Department of Civil Engineering Indian Institute of Technology, Delhi, July 1987.

Citation: Moein Zargar, et.al. "The Influence of Rock Anisotropy on the Plan of Constructions", International Journal of Constructive Research in Civil Engineering, 5(1), pp. 24-30. DOI: http://dx. doi.org/10.20431/2454-8693.0501004

Copyright: (C) 2019 Authors, This is an open-access article distributed under the terms of the Creative Commons Attribution License, which permits unrestricted use, distribution, and reproduction in any medium, provided the original author and source are credited. 